\title{
Periorale Dermatitis im Kindesalter - Klinik, Ätiopathogenese und Therapie unter Berücksichtigung eigener Erfahrungen mit dem Einsatz von $20 \%$ iger Azelainsäure-Creme
}

\author{
Perioral Dermatitis in Childhood - Clinical Features, Etiopathogenesis and Treatment with Special Reference \\ to Own Experiences with the Use of $20 \%$ Azelaic Acid Cream
}

Autoren

Institut

\section{T. Jansen, S. Grabbe}

Klinik und Poliklinik für Dermatologie, Venerologie und Allergologie der Universität Essen (Direktor: Prof. Dr. Stephan Grabbe)

\section{Bibliografie}

Dol $10.1055 / \mathrm{s}-2007-966458$

Akt Dermatol 2007; 33:

180-183 @ Georg Thieme

Verlag KG Stuttgart · New York ISSN 0340-2541

Korrespondenzadresse

Dr. Thomas Jansen

Klinik und Poliklinik für

Dermatologie, Venerologie

und Allergologie

der Universität Essen

Hufelandstraße 55

45122 Essen

thomas.jansen@medizin.uniessen.de

\section{Zusammenfassung \\ $\nabla$}

Die periorale Dermatitis ist häufig bei jüngeren Frauen, jedoch selten bei Kindern. Berichtet wird über 8 Kinder im Alter von 3-12 (durchschnittlich 7,5) Jahren mit perioraler Dermatitis, die seit 3-7 (durchschnittlich 4,9) Monaten bestand. Die Erkrankung war in typischer Morphologie zumeist sowohl perioral als auch perinasal und periokulär lokalisiert. Bei allen Patienten war der Entwicklung der Hautveränderungen eine örtliche Anwendung von niedrig- bis hochpotenten Glukokortikoiden vorangegangen. Hinweise auf eine atopische Diathese ergaben sich bei der Hälfte der Patienten. Allergologische, bakteriologische und mykologische Untersuchungen ergaben keine auffälligen Befunde. Demodex-Milben waren mit Zyanoakrylatabriss nicht nachweisbar. Bei allen Patienten erfolgte eine Lokaltherapie mit $20 \%$ iger Azelainsäure-Creme, die zweimal täglich

\section{Einleitung}

$\nabla$

Die periorale (Rosazea-artige) Dermatitis ist eine Erkrankung der Gesichtshaut, die überwiegend jüngere Frauen betrifft $[27,28]$. Bei Kindern wird sie nur selten beobachtet. In einer Zusammenstellung von 203 Fällen waren nur 15 Kinder (7,4\%) zu finden [28]. Das Auftreten der Erkrankung in dieser Altersgruppe wurde erstmals von Gianotti und Mitarbeitern [11] 1970 bei 5 Kindern im Alter von 2,5-7 Jahren in der französischen Literatur beschrieben. Mitteilungen zur perioralen Dermatitis im Kindesalter sind unter verschiedenen Bezeichnungen wie periorale Dermatitis vom Gianotti-Typ [10], Rosazea-artige Eruption der Kindheit [25], granulomatöse periorale (periorifizielle) Dermatitis der Kindheit $[4,8,15,20,30]$ oder faziale afrokaribische Eruption der Kindheit [29] zu finden. auf die betroffenen Hautpartien aufgetragen wurde. Darunter kam es nach 4-8 (durchschnittlich $5,6)$ Wochen zu einer vollständigen Abheilung aller Effloreszenzen. Bei 3 Patienten ging der Abheilung eine temporäre Exazerbation der Dermatose mit einem Maximum zwischen dem 2. und 6. Tag der Behandlung voraus, die jedoch keine zusätzliche interne Medikation erforderlich machte. Nebenwirkungen traten bei 6 Patienten bevorzugt in den ersten beiden Behandlungswochen auf. Sie bestanden in erster Linie aus einem kurzen Brennen unmittelbar nach Auftragen der Creme. Die Nebenwirkungen waren geringfügig und nahmen mit fortlaufender Behandlungsdauer ab. Sämtliche Patienten blieben in einer Nachbeobachtungsperiode von 2-8 (durchschnittlich 4,6) Monaten hauterscheinungsfrei. Die Ergebnisse dieser Studie sind ermutigend und sollten durch Plazebo-kontrollierte Studien an größeren Patientenkollektiven bestätigt werden.

Bei vielen Patienten mit dieser Erkrankung kann anamnestisch eine längerdauernde lokale Anwendung von Glukokortikoiden in Erfahrung gebracht werden [28]. Eine erfolgreiche Therapie setzt in diesen Fällen das Absetzen der Glukokortikoide voraus, das von einer temporären Exazerbation der Dermatose und allmählichen Abheilungsphase über viele Monate bis Jahre gefolgt wird. Eine Milderung der Aufflammreaktion und Beschleunigung der Rückbildung kann bei Erwachsenen durch orale Gabe von Tetrazyklinen oder -derivaten erreicht werden, diese dürfen jedoch bei Kindern vor dem 8. Lebensjahr wegen ihrer nachteiligen Effekte auf die Zahn- und Knochenbildung nicht verabreicht werden. Am häufigsten wird bei Kindern Metronidazol lokal, alleine oder in Kombination mit Erythromycin oral, eingesetzt $[2,12,16,19]$. Bei starker Ausprägung erwies sich eine Systemthe- 
rapie mit Isotretinoin (13-cis-Retinsäure) in Einzelfällen als wirksam [4].

Aufbauend auf die ermutigenden Therapieerfolge bei der perioralen Dermatitis im Erwachsenenalter [14] sollten nunmehr die Wirksamkeit und Verträglichkeit einer Lokaltherapie der perioralen Dermatitis im Kindesalter mit 20\%iger AzelainsäureCreme in einer offenen Studie untersucht werden.

\section{Ergebnisse}

$\nabla$

In die Studie wurden 8 ansonsten gesunde Kinder (3 Jungen, 5 Mädchen) im Alter von 3-12 (durchschnittlich 7,5) Jahren mit perioraler Dermatitis, die in der Hautklinik und Kinderklinik der Universität Essen rekrutiert wurden, aufgenommen (๑ Tab. 1).

Bei allen Patienten war das typische Bild einer perioralen Dermatitis mit kleinen, disseminierten oder gruppierten, follikulär gebundenen Papeln, Papulopusteln und Papulovesikeln auf diffus gerötetem, gelegentlich auch pityriasiform schuppendem Grund vorhanden. Stets war die periorale Region befallen, wobei ein schmaler erscheinungsfreier Saum um das Lippenrot auffiel. Der Gesichtsbefall beschränkte sich bei einem Patienten auf die periorale Region, bei den meisten waren Effloreszenzen auch perinasal $(87,5 \%)$ und periokulär $(37,5 \%)$ vorhanden. An subjektiven Beschwerden wurde Brennen, jedoch kein Juckreiz von der Hälfte der Patienten angegeben.

Die Hautveränderungen bestanden seit 3-7 (durchschnittlich $4,9)$ Monaten. In allen Fällen war der ambulanten Vorstellung mindestens ein erfolgloser Behandlungsversuch vorausgegangen. Bei allen Patienten war eine lokale Applikation von Glukokortikoiden unter Bevorzugung von Präparaten der Wirkstärke I (25\%) oder II (50\%) nach Niedner [16] erfolgt, daneben kamen auch Antibiotika (50\%), darunter Erythromycin oral bei 2 Patienten, und Antimykotika lokal (25\%) zum Einsatz.

Die Eigen- und Familienamnese ergab bei 4 (50\%) der Patienten Hinweise auf eine atopische Diathese und Erkrankungen des atopischen Formenkreises. Die Atopie-Pricktestung auf häufige Inhalationsallergene (Hausstaubmilbe, Hunde- und Katzenhaare, Gräser- und Baumpollen) fiel bei allen Patienten negativ aus. Die Gesamt-IgE-Bestimmung im Serum erbrachte keinen pathologischen Befund. Eine Epikutantestung (Europäische Standard- reihe) bei 4 Patienten ergab keinen Hinweis auf eine Typ-IV-Sensibilisierung. Bakteriologische Abstrichuntersuchungen aus Pustelinhalt ergaben kulturell bei 3 von 7 (42,9\%) der Patienten Staphylococcus epidermidis und bei 1 von 7 (14,3\%) der Patienten Staphylococcus aureus. Der mykologische Erregernachweis aus Schuppenmaterial und Pustelinhalt sowie Zyanoakrylatabrisse zum Nachweis von Demodex-Milben von den betroffenen Hautpartien fielen negativ aus.

Alle Patienten erhielten mit Einverständnis der Eltern eine Therapie mit 20\%iger Azelainsäure (Skinoren ${ }^{\circledR}$ )-Creme. Eine begleitende innerliche oder äußerliche Therapie war untersagt. Insbesondere durften Glukokortikoide nicht länger verwendet werden. Die Creme wurde zweimal täglich, morgens und abends, auf alle betroffenen Hautpartien aufgetragen.

Unter dieser Therapie setzte nach 4-8 (durchschnittlich 5,6) Wochen eine vollständige Abheilung aller Effloreszenzen und ein Sistieren des Brennens ein. Bei 3 (37,5\%) der Patienten ging der Abheilung eine vorübergehende Verschlechterung des Hautbefundes voraus. Pusteln entstanden neu auch auf vorher unveränderter Haut, Erythem und Schuppung nahmen zu. Dieses Aufflammphänomen erreichte ein Maximum zwischen dem 2. und 6. Tag. Während der zweiten Therapiewoche klang die Reaktion allmählich ab, und in der Folge bildeten sich die Effloreszenzen zurück. In der Nachbeobachtungsperiode von 2-8 (durchschnittlich 4,6) Monaten wurden keine Rezidive beobachtet.

Nebenwirkungen traten bei 6 (75\%) der Patienten auf, darunter etwa 5-10 min. anhaltendes Brennen sowie verstärktes Erythem oder Schuppung unmittelbar nach lokaler Applikation. Sie kamen bevorzugt in den ersten 2 Wochen der Behandlung vor, waren zumeist gering ausgeprägt und ließen mit zunehmender Behandlungsdauer nach. Bei keinem der Patienten veranlassten Nebenwirkungen einen vorzeitigen Therapieabbruch.

\section{Diskussion}

$\nabla$

Die vorliegende Arbeit bestätigt die Existenz einer Dermatose, die durch follikulär gebundene Papeln, Papulopusteln und Papulovesikel gekennzeichnet ist. Man beobachtet sie in jedem Lebensalter, selten auch im Kindesalter. Während bei Erwachsenen eine starke Präferenz für das weibliche Geschlecht auffällt, ist sie bei Kindern nicht evident.

Tab. 1 Kenndaten der in die Studie eingeschlossenen Patienten mit perioraler Dermatitis

\begin{tabular}{|c|c|c|c|c|c|c|}
\hline Pat.-Nr. & $\begin{array}{l}\text { Alter (Jahre), } \\
\text { Geschlecht }\end{array}$ & Lokalisation & Vorbehandlung & $\begin{array}{l}\text { Bestandsdauer } \\
\text { (Monate) }\end{array}$ & $\begin{array}{l}\text { Dauer bis Abhei- } \\
\text { lung (Wochen) }\end{array}$ & $\begin{array}{l}\text { Nachbeobachtung } \\
\text { (Monate), Rezidiv }\end{array}$ \\
\hline 1 & $9, w$ & Perioral, perinasal & Triamzinolonazetonid & 4 & 6 & 3 , nein \\
\hline 2 & $3, w$ & Perioral, perinasal & $\begin{array}{l}\text { Betamethason- } \\
\text { dipropionat }\end{array}$ & 5 & 5 & 5 , nein \\
\hline 3 & $10, \mathrm{~m}$ & $\begin{array}{l}\text { Perioral, perinasal, } \\
\text { periokulär }\end{array}$ & $\begin{array}{l}\text { Hydrocortison, } \\
\text { Erythromycin (oral) }\end{array}$ & 3 & 4 & 8 , nein \\
\hline 4 & $4, w$ & $\begin{array}{l}\text { Perioral, perinasal, } \\
\text { periokulär }\end{array}$ & $\begin{array}{l}\text { Mometasonfuroat, } \\
\text { Fusidinsäure (lokal) }\end{array}$ & 4 & 8 & 2 , nein \\
\hline 5 & $6, m$ & Perioral, perinasal & $\begin{array}{l}\text { Hydrocortisonacetat, } \\
\text { Erythromycin (oral) }\end{array}$ & 7 & 5 & 6 , nein \\
\hline 6 & $12, w$ & $\begin{array}{l}\text { Perioral, perinasal, } \\
\text { periokulär }\end{array}$ & $\begin{array}{l}\text { Hydrocortison-17- } \\
\text { butyrat, Clomitrazol }\end{array}$ & 6 & 4 & 4, nein \\
\hline 7 & $5, \mathrm{~m}$ & Perioral, perinasal & $\begin{array}{l}\text { Diflucortolon, } \\
\text { Ketokonazol }\end{array}$ & 5 & 7 & 6 , nein \\
\hline 8 & $11, w$ & Perioral & $\begin{array}{l}\text { Triamzinolonazeto- } \\
\text { nid, Clindamycin } \\
\text { (lokal) }\end{array}$ & 5 & 6 & 3 , nein \\
\hline
\end{tabular}


Die Morphologie und Topografie der perioralen Dermatitis im Kindesalter entspricht in vielen Punkten derjenigen im Erwachsenenalter. Neben der mikropapulösen Form, wie sie die Patienten in dieser Studie aufwiesen, gibt es eine makropapulöse und lupoide (granulomatöse) Form der Erkrankung, bei welcher die Effloreszenzen auf Glasspateldruck ein lupoides (apfelgeleeartiges) Infiltrat erkennen lassen $[12,16]$. Die lupoide Form der perioralen Dermatitis muss von einer im Kindesalter jedoch sehr seltenen Sarkoidose abgegrenzt werden. Wie bei Erwachsenen ist die Gesichtshaut beidseitig symmetrisch betroffen, wobei ein schmaler, an das Lippenrot angrenzender Bereich ausgespart bleibt. Im Kindesalter beschränkt sich der Befall häufig nicht auf die periorale Region, sondern umfasst zusätzlich den perinasalen und periokulären Gesichtsabschnitt. Sehr selten finden sich bei Kindern Effloreszenzen auch außerhalb des Gesichts am Hals oder oberen Rumpfabschnitt [13]. In dieser Studie war ein isolierter perioraler Befall nur bei einem Patienten nachweisbar, während die übrigen Patienten einen Mitbefall der perinasalen und periokulären Region zeigten. Subjektive Beschwerden waren nur bei der Hälfte der Patienten in Form von Brennen vorhanden. Ätiologie und Pathogenese der perioralen Dermatitis sind nach wie vor weitgehend ungeklärt. Selbst die Eigenständigkeit der Erkrankung wird diskutiert und eine nosologische Beziehung zur Rosazea hergestellt [26]. Obwohl beide Gesichtsdermatosen klinisch bei deutlicher Ausprägung gut differenzierbar sind und eine unterschiedliche Altersverteilung aufweisen, sind dem Dermatologen Mischbilder bekannt. Der Übergang einer perioralen Dermatitis in eine Rosazea ist keine allzu seltene Begebenheit. Das Fehlen von Teleangiektasien und konjunktivalen Reizerscheinungen bei den Patienten in dieser Studie spricht gegen das Vorliegen einer Rosazea.

In vielen, jedoch nicht in allen Fällen von perioraler Dermatitis ist ein enger zeitlicher Zusammenhang zu einer vorausgegangenen, langdauernden lokalen Anwendung von (fluorierten) Glukokortikoiden ersichtlich. Eine sorgfältige und mitunter hartnäckige Anamneseerhebung ist meist in der Lage, dies zu bestätigen. Ein bedeutender Hinweis auf einen Zusammenhang von perioraler Dermatitis und lokal angewandten Glukokortikoiden ist die eintretende Abheilung nach Absetzen der entsprechenden Präparate. In dieser Studie war bei allen Patienten ein Gebrauch von niedrig- bis hochpotenten Glukokortikoiden vor Erkrankungsbeginn eruierbar. In der Mehrzahl kamen Präparate der Wirkstärke I (25\%) oder II (50\%) nach Niedner [21] zur Anwendung. Die pathogenetische Bedeutung von Hydrocortison bleibt zweifelhaft, da dieses niedrigpotente Glukokortikoid auch erfolgreich zur Behandlung der perioralen Dermatitis eingesetzt wird $[12,16,18]$. Häufig sind es geringfügige Befunde an der Gesichtshaut, wie eine irritative Kontaktdermatitis, zu deren Behandlung Glukokortikoide verwendet werden.

Wie die lokale Anwendung von Glukokortikoiden einen derartigen Hautbefund hervorruft, wird noch diskutiert. In Betracht kommen eine direkte Glukokortikoidwirkung auf die Talgdrüsen-Follikel-Einheit oder aber eine Begünstigung einer Follikelinfektion mit Krankheitserregern, wobei vor allem Candida albicans [3] und Fusobakterien [5] eine wichtige Rolle zugeschrieben wird. Es wird auch vermutet, dass Pflegemittel, wie z. B. Feuchtigkeitscremes, durch Veränderung des Mikromilieus zu einer Proliferation ansonsten nicht-pathogener Standortflora führen könnten, die dann pathogene Eigenschaften entwickeln [9]. Allerdings waren Candida albicans, Candida species und andere Hefepilze in dieser Studie, ebenso wie in einer weiteren Untersuchung an 7 Patienten [2], bei keinem Patienten anzüchtbar.
Mikrobiologisch fand sich bei 3 Patienten Staphylococcus epidermidis, also normale Standortflora, und bei einem Patienten Staphylococcus aureus im Bereich der befallenen Hautpartien, ohne dass sich ein kausaler Zusammenhang des pathogenen Keimes zu der Erkrankung herstellen ließ. Klinisch bestand kein Anhalt für eine Impetigo contagiosa. Demodex-Milben, die wiederholt angeschuldigt wurden [24], waren mit Zyanoakrylatabriss nicht nachweisbar.

Eine Schädigung der epidermalen Barrierefunktion bei atopischer Disposition wurde als Ätiologie angesehen [6,9]. In dieser Studie ergab die Eigen- und Familienanamnese bei der Hälfte der Patienten Hinweise auf eine atopische Diathese und Erkrankungen des atopischen Formenkreises. Die Atopie-Prick-Testung auf häufige Inhalationsallergene fiel bei allen Patienten negativ aus. Die Gesamt-IgE-Bestimmung im Serum erbrachte ausschließlich altersabhängige Normalbefunde. Eine Verbindung von perioraler Dermatitis und atopischer Diathese ließ sich in dieser Studie, ebenso in einer weiteren Erhebung an 7 Patienten [2], nicht herstellen.

Gelegentlich ist eine allergische Kontaktdermatitis durch Kosmetika, Mundwässer, Kaugummis oder Schalen von Zitrusfrüchten als Ursache der perioralen Dermatitis vermutet worden [7]. In unserem Patientengut ergab die Epikutantestung (Europäische Standardreihe) jedoch keinen Hinweis auf eine Typ-IV-Sensibilisierung.

Der erste und wichtigste Schritt in der Behandlung der perioralen Dermatitis besteht im Absetzen der zuvor verwendeten Glukokortikoide. Es ist hierbei unbedingt erforderlich, die Eltern der betroffenen Kinder auf die zeitweilig zu erwartende Verschlechterung des Hautbefundes hinzuweisen. Eine Vielzahl von Medikamenten, alleine oder in Kombination, wird empfohlen, jedoch gibt es bisher keine allgemein akzeptierte Standardtherapie der perioralen Dermatitis. Angesichts der Harmlosigkeit und spontanen Rückbildungsfähigkeit der Erkrankung erscheint es sinnvoll, nach Möglichkeit eine interne Medikation zu umgehen.

Vor diesem Hintergrund könnte, aufbauend auf den positiven Therapieerfahrungen bei perioraler Dermatitis im Erwachsenenalter [14], die lokale Applikation von Azelainsäure eine mögliche Therapieoption bei perioraler Dermatitis im Kindesalter darstellen. Azelainsäure ist eine natürlich vorkommende, gesättigte, geradkettige Dikarboxylsäure mit einem Gerüst aus neun Kohlenstoffatomen (1,7-Heptandikarbonsäure), die sich in der Therapie der leichten bis mittelschweren Akne und Rosazea etabliert hat [22]. In dieser Studie setzte bei allen Patienten eine vollständige Abheilung nach 4-8 (durchschnittlich 5,6) Wochen ein. Die Dauer bis zur Abheilung war vergleichbar mit den Ergebnissen von Manders und Lucky [18], die ihre Patienten mit 0,75\% Metronidazol-Gel alleine oder in Kombination mit niedrigpotenten Glukokortikoiden (lokal) oder Antibiotika (oral oder lokal) behandelten (1-8 Wochen). Sie fiel deutlich kürzer aus als bei 7 Patienten, die allein mit $1-2 \%$ Metronidazol in Ölin-Wasser-Emulsion behandelt wurden (3-6 Monate) [2]. Bei 3 (37,5\%) der von uns behandelten Patienten ging der Abheilung eine temporäre Exazerbation der Dermatose voraus, die jedoch keine zusätzliche interne Medikation erforderlich machte. Die Creme wurde gut vertragen, wobei als häufigste Nebenwirkung bevorzugt in den ersten beiden Behandlungswochen ein kurzes Brennen unmittelbar nach lokaler Applikation zu verzeichnen war. Die Wirksamkeit der Azelainsäure bei der perioralen Dermatitis könnte auf ihre anti-inflammatorischen (Hemmung der Produktion und Freisetzung von reaktiven Sauerstoffspezies aus neutrophilen Granulozyten) [1,23] und, falls man eine patho- 
genetische Bedeutung von Mikroorganismen voraussetzt, antimikrobiellen Eigenschaften [17] zurückzuführen sein.

Angesichts der Ergebnisse dieser Pilotstudie sind weitere Plazebo-kontrollierte Studien an größeren Patientenkollektiven erforderlich, um die Wirksamkeit einer Azelainsäure-Therapie der perioralen Dermatitis im Kindesalter zu bestätigen.

\section{Abstract}

\section{Perioral Dermatitis in Childhood - Clinical Features, Etiopathogenesis and Treatment with Special Reference to Own Experiences with the Use of $20 \%$ Azelaic Acid Cream}

Perioral dermatitis, a common skin disease in young women, has been rarely reported in children. In this study, 8 children aged 3-12 (main 7.5) years suffering from perioral dermatitis for 3-7 (main 4.9) months were evaluated. The disease had developed in typical distribution and morphology, with perinasal and periocular lesions along with classical perioral lesions being present. In all patients low- to high-potency topical glucocorticosteroids had been used in the face prior to the onset of disease. Atopy was found in half of the patients. Allergological, bacteriological and mycological examinations did not reveal any pathological results. Demodex mites could not be demonstrated by skin surface biopsies. Patients were treated with $20 \%$ azelaic acid cream which was topically applied twice daily on all affected areas. Treatment led to complete resolution of skin lesions after 4-8 (mean 5.6) weeks in all patients. Transient exacerbation of skin condition with a peak between the $2^{\text {nd }}$ and $6^{\text {th }}$ day of treatment could be observed in 3 patients, but no additional internal medication was necessary. Side-effects of $20 \%$ azelaic acid cream were registered in 6 patients and were predominantly present in the first two weeks of treatment. Most patients felt short-standing burning sensation immediately after topical application. Side-effects were minimal and became more rare with ongoing treatment. No recurrences were seen within a follow-up period of $2-8$ (mean 4.6) months. The results of this study are encouraging and should be confirmed by further placebocontrolled studies in larger patient groups.

\section{Literatur}

1 Akamatsu H, Komura J, Asada Y, Miyachi Y, Niwa Y. Inhibitory effect of azelaic acid on neutrophilic functions: a possible cause for its efficacy in treating pathogenetically unrelated diseases. Arch Dermatol Res 1991; 283: $162-166$

2 Boeck K, Abeck D, Werfel S, Ring J. Perioral dermatitis in children - clinical presentation, pathogenesis-related factors and response to topical metronidazole. Dermatology 1997; 195: 235-238

3 Bradford LG, Montes LF. Perioral dermatitis and Candida albicans. Arch Dermatol 1972; 105: $892-895$
4 Brunner M, Megahed M, Hölzle E, Ruzicka T. Granulomatöse periorale Dermatitis im Kindesalter - erfolgreiche Therapie mit Isotretinoin (Roaccutan $^{\circledR}$ ). Akt Dermatol 1995; 21: 60-62

5 Buck A, Kalkoff KW. Zum Nachweis von Fusobakterien aus Effloreszenzen der perioralen Dermatitis. Hautarzt 1971; 22: 433-436

6 Dirschka T, Szliska C, Jackowski J, Tronnier H. Impaired skin barrier and atopic diathesis in perioral dermatitis. J Dtsch Dermatol Ges 2003; 1: 199-203

7 Fisher AA. Cosmetic dermatitis in childhood. Cutis 1995; 55: 15-16

8 Frieden IJ, Prose NS, Fletcher V, Turner ML. Granulomatous perioral dermatitis in children. Arch Dermatol 1989; 125: 369- 373

9 Fritsch P, Pichler E, Linser I. Periorale Dermatitis. Hautarzt 1989; 40: $475-479$

10 Georgouras K, Kocsard E. Micropapular sarcoidal facial eruption in a child: Gianotti-type perioral dermatitis. Acta Derm Venereol (Stockh) 1978; 58: 433 - 436

11 Gianotti F, Ermacora E, Benelli MG, Caputo R. Particulière dermatite péri-orale infantile. Observations sur 5 cas. Bull Soc Fr Dermatol Syphiligr 1970; 77: 341

12 Grosieux C, Stalder JF. Dermatitis périorales de l'enfant. Ann Dermatol Venereol 1997; 124: 346-350

13 Hansen KK, McTigue MK, Esterly NB. Multiple facial, neck, and upper trunk papules in a black child: childhood granulomatous perioral dermatitis with involvement of the neck and upper trunk. Arch Dermatol 1992; 128: 1396 - 1397, 1399

14 Jansen T. Azelaic acid as a new treatment for perioral dermatitis: results from an open study. Br J Dermatol 2004; 151: 933-934

15 Knautz MA, Lesher JL Jr. Childhood granulomatous periorificial dermatitis. Pediatr Dermatol 1996; 13: 131 - 134

16 Laude TA, Salvemini JN. Perioral dermatitis in children. Semin Cutan Med Surg 1999; 18: 206-209

17 Leeming JP, Holland KT, Bojar RA. The in vitro antimicrobial effect of azelaic acid. Br J Dermatol 1986; 115: 551 - 556

18 Manders SM, Lucky AW. Perioral dermatitis in childhood. J Am Acad Dermatol 1992; 27: 688-692

19 Miller SR, Shalita AR. Topical metronidazole gel $(0,75 \%)$ for the treatment of perioral dermatitis in children. J Am Acad Dermatol 1994; 31: $847-848$

20 Nguyen $V$, Eichenfield LF. Periorificial dermatitis in children and adolescents. J Am Acad Dermatol 2006; 55: 781 - 785

21 Niedner R. Glukokortikosteroide in der Dermatologie: Kontrollierter Einsatz erforderlich. Dtsch Ärztebl 1996; 52: A-2868 - A-2872

22 Passi S. Pharmacology and pharmacokinetics of azelaic acid. Rev Contemp Pharmacother 1993; 4: 441 - 447

23 Passi S, Picardo M, Zompetta C, De Luca C, Breathnach AS, Nazzaro-Porro $M$. The oxyradical-scavenging activity of azelaic acid in biological systems. Free Radic Res Commun 1991; 15: 17- 28

24 Rufli T, Mumcuoglu Y, Cajacob A, Büchner S. Demodex folliculorum: Zur Ärtiopathogenese und Therapie der Rosazea und perioralen Dermatitis. Dermatologica 1981; 162: $12-26$

25 Savin JA, Alexander S, Marks $R$. A rosacea-like eruption of children. Br J Dermatol 1972; 87: 425-429

26 Weston WL, Morelli JG. Steroid rosacea in prepubertal children. Arch Pediatr Adolesc Med 2000; 154: 62 - 64

27 Wilkinson DS. What is perioral dermatitis? Int J Dermatol 1981; 20: $485-486$

28 Wilkinson DS, Kirton V, Wilkinson JD. Perioral dermatitis: a 12-year review. Br J Dermatol 1979; 101: 245 - 257

29 Williams HC, Ashworth J, Pembroke AC, Breathnach SM. FACE - facial Afro-Caribbean childhood eruption. Clin Exp Dermatol 1990; 15: $163-166$

30 Zalaudek I, Di Stefani A, Ferrara G, Argenziano G. Childhood granulomatous periorificial dermatitis: a controversial disease. J Dtsch Dermatol Ges 2005; 3: $252-255$ 